\title{
Dynamic $B_{0}$ shimming at 7 Tesla
}

\author{
Saikat Sengupta ${ }^{a, b}$, E. Brian Welch ${ }^{a, f}$, Yansong Zhaoc ${ }^{c}$ David Foxalld Piotr Starewicz $^{\mathrm{e}}$, \\ Adam W. Anderson $a, b, f$, John C. Gore ${ }^{a, b, f}$, and Malcolm J. Avison ${ }^{a, f}$
}

Saikat Sengupta: saikat.sengupta@vanderbilt.edu; E. Brian Welch: edward.b.welch@vanderbilt.edu; Yansong Zhao: yansong.zhao@philips.com; David Foxall: david.foxall@philips.com; Piotr Starewicz: pms@rricorp.com; Adam W. Anderson: adam.anderson@vanderbilt.edu; John C. Gore: john.gore@vanderbilt.edu; Malcolm J. Avison: calum.avison@vanderbilt.edu

a Vanderbilt University Institute of Imaging Science, Vanderbilt University, Nashville, TN, 37235, USA

b Department of Biomedical Engineering, Vanderbilt University, Nashville, TN, 37235, USA

${ }^{c}$ MR Clinical Science, Philips Healthcare Inc, Cleveland, OH, 44143, USA

d Philips Medical Systems (Cleveland) Inc, Cleveland, OH, 44143, USA

e Resonance Research, Inc., Billerica, MA 01821, USA

${ }^{f}$ Department of Radiology and Radiological Sciences, Vanderbilt University, Nashville, TN, 37235, USA

\section{Abstract}

Dynamic slicewise shimming improves $\mathrm{B}_{0}$ field homogeneity by updating shim coil currents for every slice in a multi-slice acquisition, producing better field homogeneity over a volume than can be obtained by a single static global shim. The first aim of this work was to evaluate the performance of slice-wise field map based $2^{\text {nd }}$ order dynamic shimming in a human high field 7 Tesla clinical scanner vis-à-vis image based $2^{\text {nd }}$ order static global shimming. Another goal was to characterize eddy currents induced by $2^{\text {nd }}$ and $3^{\text {rd }}$ order shim switching. A final aim was to compare global and dynamic shimming through shim orders to elucidate the relative benefits of going to higher orders and to dynamic shim updating from a static shimming regime. An external hardware module was used to store and dynamically update slice-optimized shim values during multislice data acquisition. High bandwidth multislice gradient echo scans with $\mathrm{B}_{0}$ field mapping and low bandwidth single shot echo planar scans were performed on phantoms and humans using $2^{\text {nd }}$ order dynamic and static global shims. For the measurement of $2^{\text {nd }}$ and $3^{\text {rd }}$ order shim induced eddy currents, step response temporal phase changes of individual shims were measured and fit to shim harmonics spatially and to multiexponential decay functions temporally. Finally, an order wise fieldmap based comparison was performed with $1^{\text {st }}, 2^{\text {nd }}$ and $3^{\text {rd }}$ order global static shimming, $1^{\text {st }}$ and $2^{\text {nd }}$ order dynamic shimming, as well as combined $2^{\text {nd }}$ or $3^{\text {rd }}$ order global and $1^{\text {st }}$ order dynamic shim. Dynamic shimming considerably improved $\mathrm{B}_{0}$ homogeneity compared to static global shimming, both in phantoms and in human subjects, reducing image distortion and signal drop-out. The unshielded $2^{\text {nd }}$ and $3^{\text {rd }}$ order shims generated strong $\mathrm{B}_{0}$, self- and cross-term eddy fields with multiple time constants ranging from milliseconds to seconds. Field homogeneity improved with increasing order of shim, with dynamic shimming performing better than global shimming. Hybrid global and dynamic shimming approach yielded field homogeneity better than global static shims but worse than dynamic shims.

Corresponding Author: Saikat Sengupta, Vanderbilt University Institute of Imaging Science, 116121 st Avenue South, Medical Center North, AA-1105, Nashville, TN 37232-2310, Phone No: 1(615)7154360, Fax: 1(615) 322-0734, saikat.sengupta@ vanderbilt.edu. 


\section{Keywords}

Shimming; Dynamic Shimming; High Field; Eddy Currents

\section{INTRODUCTION}

In MRI, static magnetic field inhomogeneities $\left(\Delta \mathrm{B}_{0}\right)$ can arise from susceptibility differences at tissue boundaries and change with physiological processes such as respiration and cardiac motion. These field inhomogeneities cause a variety of image artifacts including geometric distortions, intensity changes and intravoxel signal losses. In spectroscopy, higher $\Delta \mathrm{B}_{0}$ causes reduced signal and broader line widths. Fast $\mathrm{T}_{2} *$ based imaging sequences such as single shot Echo Planar Imaging (SSEPI) commonly used in functional MRI are particularly sensitive to field inhomogeneities [1-3]. Susceptibility artifacts also increase with field strength as $\Delta \mathrm{B}_{0}$ varies proportionately with field. Although high field strength magnets ( 3 Tesla and greater) are being employed more commonly in order to obtain stronger MR signals, larger spectral dispersions and greater blood oxygen level dependent (BOLD) effects, the larger $\Delta \mathrm{B}_{0}$ values in practice somewhat detract from the expected benefits of high field $[4,5]$. This remains one of the unsolved technical challenges in high field imaging to date.

Compensation for field inhomogeneities has traditionally been carried out by room temperature static global shimming (GS) comprising of a set of discrete orthogonal shim coils designed to produce specific spatial field corrections to minimize field inhomogeneities [6-10]. The shim settings are usually applied in the scan preparation phase and remain constant throughout the scan. Global shims optimize $\mathrm{B}_{0}$ homogeneity over the entire volume of interest, rather than individual slices or sub-volumes. Dynamic shimming (DS) is a technique in which the slicewise shim settings are changed during the acquisition of data from multiple slices or sub-volumes. Therefore, multiple shim settings optimal for each slice or sub-volume can be applied during a single experiment, leading to better localized compensation of field inhomogeneities, than obtainable using a single global shim set [1117]. With increasing field strengths and consequently higher susceptibility artifacts, DS may be an important tool to improve image quality compared to conventional shimming techniques.

A slice-wise DS method in which the optimal first order shim currents were updated for every slice in a multislice acquisition was first proposed by Blamire et al [11] and Morrell et al [12]. DS was extended to $2^{\text {nd }}$ order by de Graaf et al in 2003[13] and Koch et al in 2006 $[14,15]$ where the eddy currents produced by rapid switching of the $2^{\text {nd }}$ order shims were characterized and compensated using a multiple time constant compensation circuit. Zhao et al. simulated DS up to $3^{\text {rd }}$ order and showed that DS can yield better optimized field homogeneity than GS [16]. DS has also been demonstrated to be effective in compensating for respiration induced $\mathrm{B}_{0}$ fluctuations in the brain [17]. In addition to slicewise shimming, DS can also be performed by optimizing $\Delta \mathrm{B}_{0}$ over parcels or blocks, rather than slices. This has been shown in simulations to achieve even greater homogeneity than slice-wise DS, as it is better suited to the rapidly changing inhomogeneity profile in the brain [18]. However, the general applicability of this approach has yet to be demonstrated experimentally.

With increased $\Delta \mathrm{B}_{0}$ values at 7 Tesla, there is a need to evaluate the benefits that may be obtained with DS over static GS. We present the results of implementing slice-wise $2^{\text {nd }}$ order DS with both high bandwidth gradient recalled echo (GRE) and low bandwidth SSEPI imaging. Eddy current fields associated with $2^{\text {nd }}$ and $3^{\text {rd }}$ order shim switching may compromise the potential improvements in $\mathrm{B}_{0}$ homogeneity provided by DS. We therefore 
measure all the $2^{\text {nd }}$ and $3^{\text {rd }}$ order shim induced eddy fields and compare the performance of a shielded and unshielded Z2 shim coil. Finally, with the purpose of elucidating the relative improvements obtained by going to higher orders and to DS from static GS methods, we compare the performance of different orders of global and dynamic shims. We present invivo comparisons of $1^{\text {st }}, 2^{\text {nd }}$ and $3^{\text {rd }}$ order GS, $1^{\text {st }}$ and $2^{\text {nd }}$ order DS, as well as combined $2^{\text {nd }}$ or $3^{\text {rd }}$ order GS and $1^{\text {st }}$ order DS.

\section{METHODS}

All studies were performed on a 7 Tesla whole body human MRI system (Philips Healthcare Inc, Cleveland Ohio, USA) with a 16 channel SENSE array receiver coil (Nova Medical, Inc, Wilmington, MA, USA) and a single channel quadrature transmit volume coil. The Philips 7T system design includes 7 unshielded $3^{\text {rd }}$ order shims (Z3, Z2X, Z2Y, Z(X2-Y2), $\mathrm{XYZ}, \mathrm{X} 3, \mathrm{Y} 3), 5$ unshielded $2^{\text {nd }}$ order shims (Z2, ZX, ZY, X2-Y2, XY) and 2 actively shielded shims (Z2Dynamic or Z2D, Z0). The shims were driven by amplifiers manufactured by Resonance Research Inc (MXH -14, \pm 10 A, RRI, Billerica, MA, USA). Each amplifier had an additional auxiliary analog input for separate additive shim drive control. First-order shimming was provided via the 3 actively shielded $1^{\text {st }}$ order gradients, controlled by the scanner $\mathrm{s}$ imaging gradient amplifiers. $2^{\text {nd }}$ order shims were controlled using an external shim switching hardware module connected to the auxiliary input of the shim system (details given below in the shim update section). Our method was based on estimating the slice-wise $\mathrm{B}_{0}$ field variations from a repeated GRE scan with a known $\Delta T E$ and analyzing the fieldmaps to calculate slice-wise shim settings for DS. Shim calculation was performed entirely in Matlab2008 ${ }^{\mathrm{TM}}$ (Mathworks Inc, Natick, MA, USA). A graphical user interface (GUI) was developed in Matlab ${ }^{\mathrm{TM}}$ and run on the console computer for expedited shim analysis. We performed studies on phantoms and human subjects. The shielded Z2D coil was used in all DS experiments. The benefits of DS were evaluated by comparing the residual $\mathrm{B}_{0}$ inhomogeneity, image distortion and signal losses to those obtained by conventional image based static GS.

\section{Phantom Experiments}

A $17 \mathrm{~cm}$ diameter, spherical "Braino" phantom (GE Medical Systems, Milwaukee, WI, USA), containing doped water was used as a test phantom. Low resolution fieldmaps using a repeated GRE sequence with a known $\triangle \mathrm{TE}(64 \times 64$ pixels, $300 \mathrm{~mm}$ FOV, 25 slices, first $\mathrm{TE} / \Delta \mathrm{TE}=4 / 1 \mathrm{~ms}$, slice thickness $/ \mathrm{gap}=2 / 2 \mathrm{~mm}$ ) were obtained in the three principal orientations, with all shims set to 0 Amps. Using a region of interest (ROI) defined by all voxels with nonzero signal in every slice, slice-wise shim coefficients up to $2^{\text {nd }}$ order were calculated using least squares minimization and fed to the external shim switching hardware module before the intended scan. For GS, a single shim set was calculated with the same regression algorithm, for the entire ROI. GRE image sets including underlying fieldmaps were then acquired at $128 \times 128$ pixel resolution using the same slice geometries, flip angle, readout bandwidth and echo times with both static and dynamic shims. Results were compared in terms of the slicewise standard deviations of the post shim fieldmaps.

\section{Human Experiments}

All human volunteers provided informed written consent and were scanned under an institutional IRB approved protocol. Low resolution fieldmaps $(64 \times 64$ pixels, $250 \mathrm{~mm}$ FOV, 25 slices, first TE $/ \Delta \mathrm{TE}=4 / 1 \mathrm{~ms}$, slice thickness $/ \mathrm{gap}=3 / 1 \mathrm{~mm}$ for axials and $3 / 2 \mathrm{~mm}$ for coronals) were obtained for 12 subjects (10 axial and 4 coronal stack orientations, same orientation not repeated for any subject) with all shims zeroed. The fieldmaps were masked using skull stripping [19] combined with an operator defined ROI to delineate the final shim region. Dynamic and global shimming coefficients were calculated and the shim switching 
module was loaded with the slicewise shim values. High resolution GRE images and fieldmaps $(256 \times 256$ pixels, same image geometry as the fieldmaps, TR/first TE/ $\Delta \mathrm{TE}=$ $820 / 20 / 1 \mathrm{~ms}$, readout bandwidth $=1349 \mathrm{~Hz} /$ pixel, SENSE acceleration factor $=2$ ) were acquired with DS and static GS conditions. The fieldmaps were evaluated by calculating the slicewise standard deviations within the ROI. In addition, the standard deviation, the range covering the central $80 \%$ of pixel values [14] (generalization of the interquartile range to the central $80 \%$, since quite often the field distribution is not normal) and the fraction of nonzero field pixels greater than $50 \mathrm{~Hz}(0.167 \mathrm{ppm}$, corresponding to $\sim 2.5$ pixel inplane displacement at $19.4 \mathrm{~Hz} /$ pixel bandwidth) were calculated within the entire multislice ROI as well as the whole brain.

To evaluate distortions arising from low bandwidth acquisitions in the presence of field inhomogeneity, axial SSEPI images were acquired with the same geometry, TR/TE = $2755 / 29 \mathrm{~ms}$, SENSE acceleration factor $\mathrm{R}=1,128 \times 128$ pixels and phase encoding bandwidth $=19.4 \mathrm{~Hz} /$ pixel. The resulting images were rigidly registered to high bandwidth GRE images to eliminate bulk shifts and then non-rigidly registered to the GRE image using the multilevel Adaptive Bases Registration algorithm (ABA) [20], which yielded complete deformation maps of the two echo planar source images quantifying the inplane pixel shifts. The registration was constrained to the phase encoding direction only. The standard deviation of the pixel shift deformation map was calculated to yield a measure of the amount of distortion in the echo planar images. This method gives a more complete picture of distortion, based on measuring the mutual information between images, thereby capturing distortion information from the entire image as compared to only the edges or a single profile.

To investigate the relative benefits of increasing orders of global and dynamic shimming and also to identify shimming techniques that may yield improvements on static GS without the need for dynamic higher order shim switching hardware, a separate set of human head fieldmapping experiments were conducted. In addition to standard global and dynamic shimming, a hybrid shim approach was employed in which $1^{\text {st }}$ order shims were switched dynamically on top of a static global $2^{\text {nd }}$ or $3^{\text {rd }}$ order shim (GS2DS1 or GS3DS1). Shim calculation for this technique followed the standard GS calculation described above with residual slicewise first order field corrections calculated and added for each slice dynamically during the scan using the imaging gradients. Such a technique required no additional scans, time penalty and was expected to yield better shim performance than a static global shim, without the use of any higher order dynamic shim switching hardware. 7 shim types were performed including $1^{\text {st }}, 2^{\text {nd }}$ and $3^{\text {rd }}$ order GS (GS1, GS2 GS3), $1^{\text {st }}$ and $2^{\text {nd }}$ order DS (DS1, DS2) as well as combined $2^{\text {nd }}$ or $3^{\text {rd }}$ order GS and $1^{\text {st }}$ order DS (GS2DS1, GS3DS1). Only DS2 required the higher order dynamic shim switching hardware. Whole brain GRE field mapping experiments were carried out in the axial and coronal orientations (axial $\mathrm{n}=7$, coronal $\mathrm{n}=5,128 \times 128$ pixels, 25 slices TR/first TE/ $\Delta \mathrm{TE}=320 / 10 / 1 \mathrm{~ms}$, slice thickness/gap $=3 / 1 \mathrm{~mm}$ for the axials, $3 / 2 \mathrm{~mm}$ for the coronal, $250 \mathrm{~mm}$ FOV) for each of the 7 shim types. Fieldmaps were analyzed for shim performance with the metrics described above calculated over the whole brain. To evaluate the significance of differences in the $2^{\text {nd }}$ and higher order shims (GS2, GS2DS1, GS3, GS3DS1 and DS2) a Repeated Measures Analysis of Variance (ANOVA) was performed on the 5 groups, with post hoc paired $t$ tests at $\alpha=0.05$ (SPSS19, IBM, Somers, NY, USA). A conservative Bonferroni correction was applied on the post hoc tests to account for the multiple testing. We did not include the $1^{\text {st }}$ order shims in the test as most scanners already possess and routinely use $2^{\text {nd }}$ order static shims at the least if not $3^{\text {rd }}$ order. 


\section{Shim Calculation}

When calculating single slice shim coefficients ignoring slice thickness, different shim harmonics can reduce to the same functional form and the assumption of independence between shim terms no longer holds, leading to degeneracy [14,21]. An analytical solution to this degeneracy problem was presented in detail by Koch et al [14], where the complete shim set is reduced to a subset of linearly independent shims for any slice orientation. Briefly, in order to extract the non-degenerate shim set, a coordinate transformation from the magnet coordinate system, i.e., $x, y, z$ to the rotated slice coordinate system or $x^{\prime}, y^{\prime}, z^{\prime}$ is employed. If $\alpha, \beta$, and $\gamma$ represent the angular rotations of the imaging plane about the three magnet coordinate axes $x, y, z$, the new coordinates are related to the original coordinates by the transformation matrix:

$$
\left[\begin{array}{c}
x^{\prime} \\
y^{\prime} \\
z^{\prime}
\end{array}\right]=\left[\begin{array}{ccc}
\operatorname{Cos}(\beta) \operatorname{Cos}(\gamma) & \operatorname{Cos}(\alpha) \operatorname{Sin}(\gamma)+\operatorname{Sin}(\alpha) \operatorname{Sin}(\beta) \operatorname{Cos}(\gamma) & \operatorname{Sin}(\alpha) \operatorname{Sin}(\gamma)-\operatorname{Cos}(\alpha) \operatorname{Sin}(\beta) \operatorname{Cos}(\gamma) \\
-\operatorname{Cos}(\beta) \operatorname{Sin}(\gamma) & \operatorname{Cos}(\alpha) \operatorname{Cos}(\gamma)-\operatorname{Sin}(\alpha) \operatorname{Sin}(\beta) \operatorname{Sin}(\gamma) & \operatorname{Sin}(\alpha) \operatorname{Cos}(\gamma)+\operatorname{Cos}(\alpha) \operatorname{Sin}(\beta) \operatorname{Sin}(\gamma) \\
\operatorname{Sin}(\beta) & -\operatorname{Sin}(\alpha) \operatorname{Cos}(\beta) & \operatorname{Cos}(\alpha) \operatorname{Cos}(\beta)
\end{array}\right]\left[\begin{array}{c}
x \\
y \\
z
\end{array}\right]
$$

Equating the through plane coordinate, $z$ ' yields a set of relations between the magnet coordinates true for that slice. A reduced shim set can then be obtained by inserting the relationships between these magnet coordinate directions into the shim functions and removing functional redundancies from the shim set. The fieldmap data for this slice are fit only to the reduced shim set to obtain in-plane correction values. The through-plane fields for usual slice widths are approximated as linear gradients. The projections of the field gradient normal to the slice onto the principal gradient directions give the through-plane shim corrections. These corrections add up to the required field gradient in the direction normal to the plane, while cancelling out in-plane, preserving the in-plane field.

For our studies, the non-degenerate shim set was obtained from the full set based on the slice orientation. This set was then used to calculate the slice-wise in-plane shim coefficients by multiple linear least squares fitting. A residual fieldmap was simulated using only the calculated in-plane shim values. The through-plane linear gradient corrections for each slice were then calculated by fitting the data from this simulated fieldmap in sets of three slices centered on the target slice. All the shim regressions were constrained to \pm 10 Amps, the maximum current rating of the shim channels.

Applying higher order shims to compensate for inhomogeneity for a slice at an offset location generates additional lower order terms [22]. The corrections required to cancel this effect of imaging slice displacement were calculated explicitly and added to the basic set of shim corrections, which assume that the slice is at isocenter. For oblique slices, the inverse of Eq. (1) was used to map the slice data points to the magnet coordinate system and calculate the shims. The calculated shim values were converted to current values using previously measured calibration constants. Static cross term corrections up to $2^{\text {nd }}$ order retrieved from the calibration were also calculated and added to the shim values. An exceptionally large static coupling was observed between the unshielded Z2 shim and main magnetic field.

The slicewise f0 shifts associated with the dynamic addition of the shims were corrected in every slice using the spectrometer s f0 setting. In addition to the static $\mathrm{f} 0$ changes, dynamic eddy current related $\mathrm{f} 0$ changes also originate from unshielded shim switching. The above procedure does not correct for the eddy current induced f0 shifts. These changes add to the static f0 changes and decay with multiple time constants. Compensation for these continuously changing f0 offsets requires Z0 shim waveform compensation. We have not 
included shim eddy current compensation in this work, as it requires additional, multiple waveform shaping circuits for each shim channel.

\section{Shim Update}

The first order slice-wise shim coefficients and frequency offsets were calculated and written into text files on the console computer. During scanning, these slice-wise shims were read into an array in the pulse program and applied using the $\mathrm{X}, \mathrm{Y}, \mathrm{Z}$ gradient controls before every slice excitation.

Dynamic update of $2^{\text {nd }}$ order shims was performed using a separate shim control hardware module. (Load \& Go Real Time Shims RTS' MXV 14/4, RRI, Billerica, MA, USA). The RTS system is a multi-channel small signal driver providing pre-programmed signals to the scanner shim amplifier system to allow dynamic shimming operations. It consisted of a solid state hard drive computer, RS232 serial communication ports and digital to analog converters (16 bit DAC, 4 DACs per channel, 15 channels) controlled by the computer. The GUI on the console computer communicated with the RTS via an RS232 serial connection, with custom made commands to send in the ordered shim values. The RTS did not provide controls for the X3 and Y3 channels and had separate outputs for Z2 and Z2D. The module was connected to the auxiliary input of the shim unit via an analog connection.

During the scan, slice specific shim values preloaded into the RTS were applied on receipt of a trigger pulse from the spectrometer clock. These $5 \mu$ s long trigger pulses were preprogrammed into the pulse sequence before every excitation pulse. The time taken by the module to acknowledge the trigger, update the DACs and load the shim file was $\sim 5-8 \mathrm{~ms}$ and the time required for shim amplifier current output stabilization was an additional $\sim 3 \mathrm{~ms}$ (rated shim channel rise time, $\sim 1 \mathrm{~ms}+$ settling time, $\sim 2 \mathrm{~ms}$ ), making the total time required for output current update of all shims $\sim 10 \mathrm{~ms}$. This time delay was built in the scan sequence. Figure 1 shows the schematic of the scanner shim and gradient system, the integration of the RTS accessory and auxiliary computer.

\section{Eddy Current Measurements}

Rapid switching of unshielded shims can generate severe eddy currents in the conducting structures of the magnet, which produce eddy current fields that degrade image quality. The first order shims driven by the gradient channels, were actively shielded, have pre-emphasis and therefore produce only minimal eddy currents. We characterized the eddy currents produced by the switching of $2^{\text {nd }}$ and $3^{\text {rd }}$ order shims using the method of Terpstra et al [23] adapted for shims instead of first order gradients. The X3 and Y3 shim eddy currents were measured using a trial version of the next generation RTS.

A bar was excited in the spherical "braino" phantom, along the direction where the shim of interest produced the maximum field (e.g., $45^{\circ}$ about the $\mathrm{Z}$ axis for a $\mathrm{XY}$ shim). A $1 \mathrm{~A}$ test shim pulse was turned on for 5 seconds and then turned off. Phase data were collected after waiting exponentially increasing amounts of time in every TR, with phase encoding gradient set to zero. (FOV $300 \mathrm{~mm}$, bar length $/$ width $=300 / 20 \mathrm{~mm}$, TE $=15 \mathrm{~ms}$, Mixing Time $=100$ $\mathrm{ms}$, Time after pulse switch off $=5+$ phase encode number ${ }^{1.8} \mathrm{~ms}, \mathrm{TR}=3000 \mathrm{~ms}$ ). Phase measurement was performed once with the shim pulsed and once without, for reference data. Phase along the excited bar for every time point, were obtained by unwrapping the phase data spatially and subtracting the reference phase. Every time point phase data along the bar was then fit to a combination of the switched shim and lower order shims i.e. $0^{\text {th }}, 1^{\text {st }}, 2^{\text {nd }}$ orders for the $2^{\text {nd }}$ order shims and $0^{\text {th }}$ through $3^{\text {rd }}$ order, for the $3^{\text {rd }}$ order shims. Eddy field decay amplitudes and time constants for each of these field harmonics were then determined 
by fitting the results of the first fit for each of these harmonics to a sum of 3 exponential decay function representing short, medium and long time constants.

Imaging experiments were also performed initially with the unshielded and shielded $\mathrm{Z} 2$ coils for comparison purposes (single shot gradient echo EPI, 9 slices, FOV $=250 \times 250 \mathrm{~mm}, 192$ $\times 192$ pixels, TR/TE $=2000 / 26 \mathrm{~ms}$, SENSE acceleration factor $=3$ ). The Z2D coil was then employed in all our subsequent studies (including all the phantom and human studies described above).

\section{RESULTS}

\section{Phantom Results}

Figure 2 compares slice-wise $\mathrm{B}_{0}$ inhomogeneity following $2^{\text {nd }}$ order GS and DS in the spherical phantom. The standard deviation of the residual $\Delta \mathrm{B}_{0}$ expressed in ppm was lower for DS than GS fieldmaps, for all slices and orientations, with the largest improvements observed in the coronal slices. Figure $2 \mathrm{a}$ shows the first, middle $\left(13^{\text {th }}\right)$ and last $\left(25^{\text {th }}\right)$ slice fieldmaps for the axial orientation, illustrating the improvements made by DS. Figure $2 \mathrm{~b}$ displays the simulated fieldmaps for the corresponding slices shown in Figure 2a. For all slices, the experimental and simulated fieldmaps matched closely validating the accuracy of the DS and GS implementations. Minor differences may arise from small errors in shim calibration and eddy currents in DS. The residual maps also show strong $3^{\text {rd }}$ order field variations, as expected. Figure $2 \mathrm{c}$ shows the slicewise residual field standard deviation values, in all the three orientations.

\section{Human Results}

Figures $3 \mathrm{a}$ and $3 \mathrm{~b}$ display fieldmaps of five axial and coronal slices from different subjects. The shim ROI is the box shown in the $1^{\text {st }}$ DS slice image, propagated to all the slices. Most of the slices show large reduction in field variation. Figures $3 \mathrm{c}$ and $3 \mathrm{~d}$ show the slice-wise standard deviation of the field within the ROIs. We observed larger gains in fieldmap homogeneity with DS vis-à-vis GS in the inferior axial slices and the anterior coronal slices. In these locations, higher pre-shim field inhomogeneity variation was observed owing mainly to large susceptibility gradients caused by air-bone-tissue interfaces of the frontal sinuses and the ear canals. Figure $4 \mathrm{a}$ and $4 \mathrm{~b}$ give estimates of the field flattening in the entire multislice shim ROI and the whole brain after DS and static GS in terms of field standard deviation, the central $80 \%$ pixel value range and the fraction of nonzero field value pixels greater than $50 \mathrm{~Hz}$ (Mean +/- 95\% confidence interval). As shown, DS improved all three measures of field inhomogeneity over both the shim ROI and the whole brain.

Results from SSEPI are shown in Figure 5. Five axial slices with the image outlines from corresponding high bandwidth structural GRE images, superimposed for reference are displayed. Globally shimmed images have larger pixel shifts and extend further beyond and within the reference GRE outline. Areas of distortion correspond closely to their underlying fieldmap values (Fig 3a), with high positive field deviations causing pixel shifts to the left and vice versa (phase encoding was left - right). DS reduced distortions considerably, especially in the frontal areas of the brain, which have high distortion when static $2^{\text {nd }}$ order GS is employed. Figures $6 a, b$ and c show registration results for one slice (slice 15 in Fig 3a) with the corresponding GS and DS fieldmaps. Figure 6d shows the slice-wise standard deviation of the final pixel displacement maps, a measure of distortion obtained from the registration of the EPI images (phase encoding bandwidth $=19.4 \mathrm{~Hz} /$ pixel) to the high bandwidth low distortion GRE images. DS echo planar images had smaller pixel displacements in the phase encoding direction compared to GS images for all slices. The 
deformation maps indicating pixel shifts correlate spatially to the underlying fieldmaps validating the method and giving a good measure of distortion.

Examples of differences in intravoxel signal losses caused by field gradients in dynamically and globally shimmed axial images are shown in Figure 7. Larger differences in signal levels were observed in the inferior slices of the brain, possibly owing to higher field gradients caused by the frontal sinus and the auditory canals that were better compensated with DS than GS. DS images show signal recovery in multiple areas where signal has been lost due to residual inhomogeneities in the GS case.

Figure 8 shows the results of shim order comparison in axial and coronal orientations. As predicted, the shim performance measured by the volume standard deviation, central $80 \%$ range and the number of pixels $>50 \mathrm{~Hz}$ over the whole brain improved with increasing shim order for both axial and coronal orientations. The repeated measures ANOVA revealed significant differences in the set of 5 shims types tested $(p<0.01)$. Additional Post-hoc paired $t$ tests with Bonferroni corrections revealed significant differences between individual shim types, as displayed in Figure 8.

Field homogeneity improved monotonically with increasing shim order for both GS and DS as expected, although the improvements in going from $1^{\text {st }}$ to $2^{\text {nd }}$ order were considerably higher that those in going from $2^{\text {nd }}$ to $3^{\text {rd }}$ order. In both orientations $2^{\text {nd }}$ order DS performed the best even when compared to higher order static shims GS3 and GS3DS1, while $1^{\text {st }}$ order GS had the most residual inhomogeneity. This result underscored the potential benefits of higher order DS at 7 Tesla. Greater improvements in homogeneity were observed in going from $1^{\text {st }}$ to $2^{\text {nd }}$ orders in the coronal orientation than the axial orientation for both DS and GS. In the coronal orientation, $2^{\text {nd }}$ order GS performed better than $1^{\text {st }}$ order DS according to all three measures. In the axial orientation, the differences were not significant.

Importantly, the combined global and dynamic shimming techniques GS2DS1 and GS3DS1 implemented without any higher order shim switching hardware improved field homogeneity over $2^{\text {nd }}$ and $3^{\text {rd }}$ order GS respectively. Greater improvements were observed in the axial orientation. The highest improvements were observed in the inferior axial slices where strong linear residual fields post $2^{\text {nd }}$ order GS (Fig 3a, c) were compensated for by the slicewise additional $1^{\text {st }}$ order dynamic corrections. Similar improvement was not achieved in the coronal orientation indicating the presence of primarily higher order residual fields post GS.

\section{Eddy Current Measurement Results}

Eddy currents from shielded and pre-emphasized first order gradients were not probed as gradient switching for those terms are already compensated. Table 1 gives the amplitudes and time constants from all the shims in our system, which can be used to calibrate eddy current compensation circuits. Most $2^{\text {nd }}$ order shims were seen to produce decaying $\mathrm{B}_{0}$ and self eddy fields following shim switch, while $\mathrm{XY}$ and $\mathrm{X} 2-\mathrm{Y} 2$ shims produced additional minor $1^{\text {st }}$ order fields. Of the $2^{\text {nd }}$ order shims, the unshielded $\mathrm{Z} 2$ coil produced the largest $\mathrm{B}_{0}$ variations per $\mathrm{Hz} / \mathrm{cm}^{2}$ of shim field. Figure 9a shows an example of a XY shim eddy field decaying with time after a step change of $\pm 1 \mathrm{Amp}\left( \pm 3.13 \mathrm{~Hz} / \mathrm{cm}^{2}\right)$. Third order shims produced smaller $\mathrm{B}_{0}$ variations than $2^{\text {nd }}$ order shims, but they coupled very strongly with $1^{\text {st }}$ order gradients, with long time constants ranging from 100s of milliseconds to a few seconds. Figure $9 \mathrm{~b}$ shows the $\mathrm{X}$ gradient eddy decay observed after 1 Amp switching of the $\mathrm{Z} 2 \mathrm{X}$ shim, illustrating the long time required for the gradient field to decay. The longest time constants for the $3^{\text {rd }}$ order shims were found to be, in general, longer than those for the $2^{\text {nd }}$ order shims, except for the unshielded Z2 coil. Many of the eddy fields were also observed to have reversing polarities as seen in figures $9 \mathrm{~b}$ and $9 \mathrm{~d}$. Some of the 
measurements showed a very rapidly decaying initial component with time constant less than a millisecond. However, these signals were not perfectly reproducible, most likely owing to the slight DAC update time variation. With better control of the shim ramp and DAC update times, it might be possible to characterize these signals more accurately.

The unshielded $\mathrm{Z} 2$ shim coupled strongly to $\mathrm{B}_{0}$, producing large long time constant variations in the main magnetic field. Figures $9 \mathrm{c}$ and $9 \mathrm{~d}$ show the decay of the self eddy field and $B_{0}$ when $Z 2$ and $Z 2 D$ coils are switched off from a value of $-3 \mathrm{~Hz} / \mathrm{cm}^{2}$ ( 1.5 and 5 Amperes for the Z2 and Z2D coil respectively). The Z2D coil generated a smaller and faster decaying $\mathrm{B}_{0}$ and self eddy fields compared to the $\mathrm{Z} 2$ coil, as expected. Figure $9 \mathrm{e}$ shows dynamically shimmed SS EPI images obtained with the same imaging parameters and shim correction fields, using the unshielded and shielded Z2 coils. The images with the Z2D coil show lesser ghosting artifacts than those using the $\mathrm{Z} 2$ coil, which produced virtually unusable images.

\section{DISCUSSION}

We have implemented higher order dynamic slice-wise $\mathrm{B}_{0}$ shimming on a 7 Tesla whole body MRI system, by incorporating an external hardware module to store shim values and access the shim supply, in addition to standard communication to the shim unit from the spectrometer. We have also presented a comparison of shimming techniques including hybrid approaches which do not require shim switching hardware, to evaluate the relative benefits of going to higher orders of shimming and to DS from GS. Finally we have presented $2^{\text {nd }}$ and $3^{\text {rd }}$ order shim induced eddy current measurements.

Using the RTS module allowed us to access the shim supply and partly circumvent the hardware limitations that make DS difficult. The shim amplifiers however had relatively long settling times (+/- $15 \mathrm{ppm}$ of nominal full scale within $2 \mathrm{~ms}$ rated) when connected to the inductive shim coil loads. In addition, the current RTS module added $5-8 \mathrm{~ms}$ of variable DAC update time. As a result, a $10 \mathrm{~ms}$ time delay had to be added after each trigger before MR signal excitation, which added considerable time to the GRE scans. This settling time also depended on the magnitude of the shim switch. Therefore, one might be able minimize the time penalty in the GRE DS by extending the delay for the largest shim switches while minimizing the delays for the other switches. Our system did not allow extending the ramp time of the shims which may help to reduce the amplifier settling noise, very short time constant eddy currents and shim acoustic noise [14]. A final goal in this regard would be to minimize DAC update and amplifier settling times and providing fast, flexible control of the shims via the spectrometer and software objects in the pulse programming environment. In general, to minimize amplifier settling and eddy current effects, the shim switch can be made as much before excitation as possible, for instance, just after the acquisition of the preceding slice.

DS showed considerable improvements over static GS, reflected in terms of fieldmap homogeneity, geometric distortion and intravoxel signal loss in images. This signal recovery is critical in BOLD fMRI where the activation is associated with around 5-10\% signal increase, observed at 7 Tesla [24]. Although DS does not enable complete signal recovery as it is inherently limited by the spherical harmonics and shim coil strengths, it can yield improved image quality over static GS methods. DS yields the greatest benefits over GS when the imaging volume is relatively large and the inhomogeneity changes substantially over the volume. For smaller volumes with more uniform homogeneity profiles, the improvements obtained are relatively small. We have compared DS to an image based static GS instead of a projection based shimming method, such as FASTMAP [10]. In our experience, projection based methods did not perform well in whole brain studies at 7 Tesla, 
when the ROI was extended into the frontal and inferior regions of the brain. The reason for this was not investigated, although we suspect that signal voids in these areas of the brain cause errors in estimating the shims. Also, when calculating shim coefficients, fieldmap thresholding is an often overlooked but critical step, which can otherwise lead to large errors and increased variation in shim values. For example, in the brain fieldmaps, it is essential to exclude the scalp field information. It was also found necessary to constrain the shim calculation to limits based on shim amplifier ratings, especially for the weaker Z2D. Constraining the shim calculation also reduced slice to slice variations in shim values, leading to smoother shim transitions and reduced eddy current related artifacts.

We employed the method outlined by Koch et al [14] for calculation of the slicewise shims. A different approach for tackling the degeneracy problem in DS considers the fieldmap data from slices on either side in addition to the slice in question in the regression algorithm. However, optimization to three slices rather than only the target slice can reduce the local optimization benefit of DS. Although such an approach might possibly give worse in-plane compensation, it also would yield better through-plane compensation than the first method, in which, through-plane correction is estimated to be linear. In the first method, the assumption of slices having linear though-slice inhomogeneity profile will break down as we move to thicker slices.

In our measurements, higher order unshielded shims produced strong $\mathrm{B}_{0}$, self or cross term eddy fields, with multiple decay time constants varying from milliseconds to seconds. Of the 2nd order shims, the $\mathrm{Z} 2$ unshielded channel had the strongest eddy currents with the longest time constants which agree with Koch et al [14]. However, the Z2 harmonic was observed to contribute significantly to the field corrections, rendering it indispensable. In the imaging experiments, the tesseral second order shims most often operated with inter-slice switches of much lesser than $10 \%$ of maximum (1 Amp), both on humans and phantoms leading to minimal eddy field effects from those unshielded shims. The presence of an actively shielded Z2 shim coil in our scanner was absolutely critical in reducing eddy currents and image artifacts in SS EPI. Our results with eddy current measurement and imaging comparing Z2 and Z2D precluded the use of an unshielded Z2 coil in DS (Figure 9). Careful thresholding of the fieldmaps to remove erroneously unwrapped field regions, spike noise pixels before calculating the inplane and the through plane shims, removing scalp and extraneous field information and constraining the shims helped considerably in reducing the noise in inter-slice shim variations. These steps in conjunction with using the Z2D shim coil were critical in allowing SS EPI images without considerable ghosting artifacts. The use of a Z2D coil however came at the cost of reduced shim strength, $-0.58 \mathrm{~Hz} / \mathrm{cm}^{2} / \mathrm{A}$ vs $-1.97 \mathrm{~Hz} /$ $\mathrm{cm}^{2} / \mathrm{A}$ for the unshielded Z2 coil. In future implementations of DS, both the Z2 coils will be used simultaneously. The unshielded coil will provide a constant value to which slicewise $\mathrm{Z} 2 \mathrm{D}$ changes will be added, increasing the $\mathrm{Z} 2$ dynamic range. It is important to note that using the Z2D coil does not completely eliminate eddy fields from Z2 switching, especially in the $\mathrm{B}_{0}$ channel as observed in our measurements. Improvements in shim coil shielding techniques and addition of shim eddy current compensation will improve DS in the future. The eddy current values measured here may be used as initial estimates for adjusting a compensation system. The pre-emphasis shim currents will produce their own eddy current fields, which have to be compensated in an iterative manner. Our measurements did not separate any non-eddy current effects that can also result in field changes i.e., current nonconstancy effects or vibration induced emfs that are not compensated for by the shim amplifiers [25].

Third order shims, especially Z3, Z2X and Z2Y, were seen to produce very strong first order fields with long time constants in the $\mathrm{Z}, \mathrm{X}$ and $\mathrm{Y}$ directions respectively, when the shims were switched dynamically. The magnitudes of these first order fields decayed with time 
and scaled with the switch magnitude of the $3^{\text {rd }}$ order shims. These fields were different from static interactions between the $3^{\text {rd }}$ and $1^{\text {st }}$ order shims, which were, themselves quite large (e.g. $-22.57 \mathrm{~Hz} / \mathrm{cm}$ of X per Hz/cm ${ }^{3}$ of Z2X, $-7.08 \mathrm{~Hz} / \mathrm{cm}$ of $\mathrm{Y}$ per $\mathrm{Hz} / \mathrm{cm}^{3} \mathrm{Z} 2 \mathrm{Y}$ ). The absence of an effective compensation system for these first order decaying eddy fields hindered us from implementing $3^{\text {rd }}$ order DS. Compensation of these strong linear eddy fields however might entail delicate adjustments of the gradient signal channels.

One method of reducing eddy current effects might be to switch shims about a global optimum value, such that the amplifier returns to that value after acquisition for every slice. With such a pattern of shim switching, it might be possible to generate equal and opposite polarity eddy currents from the switching, to cancel the long time constant eddy currents from the rising edge [26]. Such an approach will however require additional shim switches that could potentially introduce more noise in the shim waveforms. Alternatively, is possible to add only first order shims dynamically with each slice on top of a static global $2^{\text {nd }}$ or $3^{\text {rd }}$ order volume shim as presented here. This approach did not require extra hardware like the RTS system and can be easily implemented on most scanners. The results obtained demonstrate that this technique can be used to provide at least partial benefits of DS without the complicating factors of dynamic shim control and eddy currents. The benefits over GS however will vary with the slice orientation and the nature of the inhomogeneities.

\section{CONCLUSIONS}

Dynamic slice-wise shimming has been implemented on a 7 Tesla high field imaging system and has been shown to produce better field homogeneity, lesser distortion and lesser intravoxel signal losses compared to static GS method in phantoms as well as human whole brain studies. The results demonstrate the feasibility and benefits of DS in high field imaging. The use of a shielded Z2 coil was found to be necessary for obtaining ghosting artifact free images with DS. $2^{\text {nd }}$ and $3^{\text {rd }}$ order unshielded shims were seen to produce long time constant eddy currents of self and lower orders. $2^{\text {nd }}$ order DS was found to yield higher field homogeneity than even $3^{\text {rd }}$ order static GS while a combination of $2^{\text {nd }}$ and $3^{\text {rd }}$ order global and $1^{\text {st }}$ order dynamic shims was also found to improve field homogeneity over $2^{\text {nd }}$ and $3^{\text {rd }}$ order GS. Further work in optimizing shim switching strategies, shim coil shielding, shim eddy current compensation and software control of shims is needed for a more robust implementation of DS to maximize its benefits.

\section{Acknowledgments}

The authors thank Chuck Nockowski and Piotr Diduch for their contributions. The work was funded by NIH Grant No RO1EB000461

\section{References}

1. Schenck JF. The role of magnetic susceptibility in magnetic resonance imaging: MRI magnetic compatibility of the first and second kinds. Med Phys. 1996; 23:815-850. [PubMed: 8798169]

2. Jezzard P, Clare S. Sources of distortion in functional MRI data. Hum Brain Mapp. 1999; 8:80-85. [PubMed: 10524596]

3. Truong T, Chakeres DW, Beversdorf DQ, Scharre DW, Schmalbrock P. Effects of static and radiofrequency magnetic field inhomogeneity in ultra-high field magnetic resonance imaging. Magn Reson Imaging. 2006; 24:103-112. [PubMed: 16455399]

4. Uğurbil K, Hu X, Chen W, Zhu XH, Kim SG, Georgopoulos A. Functional mapping in the human brain using high magnetic fields. Philos Trans R Soc Lond B Biol Sci. 1999; 354:1195-1213. [PubMed: 10466146]

5. Dydak U, Schar M. MR Spectroscopy and Spectroscopic Imaging: Comparing 3.0 T versus $1.5 \mathrm{~T}$. Neuroimag Clin N Am. 2006; 16:269-283. 
6. Golay MJE. Field Homogenizing Coils for Nuclear Spin Resonance Instrumentation. The Review of Scientific Instruments. 1958; 29(4):313-315.

7. Hoult DI, Chmurny GN. The Ancient and Honourable Art of Shimming. Concepts in Magnetic Resonance. 1990; 2:131-149.

8. Schneider E, Glover G. Rapid in vivo proton shimming. Magn Reson Med. 1991; 18:335-347. [PubMed: 2046515]

9. Webb P, Macovski A. Rapid, fully automatic, arbitrary-volume in vivo shimming. Magn Reson Med. 1991; 20:113-122. [PubMed: 1943653]

10. Gruetter R. Automatic localized in vivo adjustment of all first- and second-order shim coils. Magn Reson Med. 1993; 29:804-811. [PubMed: 8350724]

11. Blamire AM, Rothman DL, Nixon T. Dynamic shim updating: a new approach towards optimized whole brain shimming. Magn Reson Med. 1996; 36:159-165. [PubMed: 8795035]

12. Morrell G, Spielman D. Dynamic shimming for multi-slice magnetic resonance imaging. Magn Reson Med. 1997; 38:477-483. [PubMed: 9339449]

13. De Graaf RA, Brown PB, McIntyre S, Rothman DL, Nixon TW. Dynamic shim updating (DSU) for multislice signal acquisition. Magn Reson Med. 2003; 49:409-416. [PubMed: 12594742]

14. Koch KM, McIntyre S, Nixon TW, Rothman DL, de Graaf RA. Dynamic shim updating on the human brain. J Magn Reson. 2006; 180:286-296. [PubMed: 16574443]

15. Koch KM, Sacolick LI, Nixon TW, McIntyre S, Rothman DL, de Graaf RA. Dynamically shimmed multivoxel $1 \mathrm{H}$ magnetic resonance spectroscopy and multislice magnetic resonance spectroscopic imaging of the human brain. Magn Reson Med. 2007; 57:587-591. [PubMed: 17326186]

16. Zhao Y, Anderson AW, Gore JC. Computer simulation studies of the effects of dynamic shimming on susceptibility artifacts in EPI at high field. J Magn Reson. 2005; 173:10-22. [PubMed: 15705507]

17. Van Gelderen P, de Zwart JA, Starewicz P, Hinks RS, Duyn JH. Real-time shimming to compensate for respiration-induced B0 fluctuations. Magn Reson Med. 2007; 57:362-368. [PubMed: 17260378]

18. Poole M, Bowtell R. Volume Parcellation for improved dynamic shimming. Magn Reson Mater Phy. 2008; 21:31-40.

19. Smith SM. Fast robust automated brain extraction. Human Brain Mapping. 2002; 17:143- 155. [PubMed: 12391568]

20. Rohde GK, Aldroubi A, Dawant BM. The Adaptive Bases Algorithm for Intensity-Based Nonrigid Image Registration. IEEE Trans Med Imag. 2003; 22:1470- 1479.

21. Shen J. Effect of degenerate spherical harmonics and a method of automatic shimming of oblique slices. NMR Biomed. 2001; 14:177-183. [PubMed: 11357182]

22. Hoult DI. "Shimming” on spatially localized signals. J Magn Reson. 1987; 73:174-177.

23. Terpstra M, Andersen PM, Gruetter R. Localized eddy current compensation using quantitative field mapping. J Magn Reson. 1998; 131:139-143. [PubMed: 9533916]

24. Yacoub E, Shmuel A, Pfeuffer J, Van De Moortele PF, Adriany G, Andersen P, Vaughan JT, Merkle H, Ugurbil K, Hu X. Imaging brain function in humans at 7 Tesla. Magn Reson Med. 2001; 45:588-594. [PubMed: 11283986]

25. Nixon TW, McIntyre S, Rothman DL, de Graaf RA. Compensation of gradient-induced magnetic field perturbations. J Magn Reson. 2008; 192:209-217. [PubMed: 18329304]

26. Wider G, Dotsch V, Wuthrich K. Self-compensating pulsed magnetic-field gradients for short recovery times. J Magn Reson Series A. 1994; 108:255-258. 


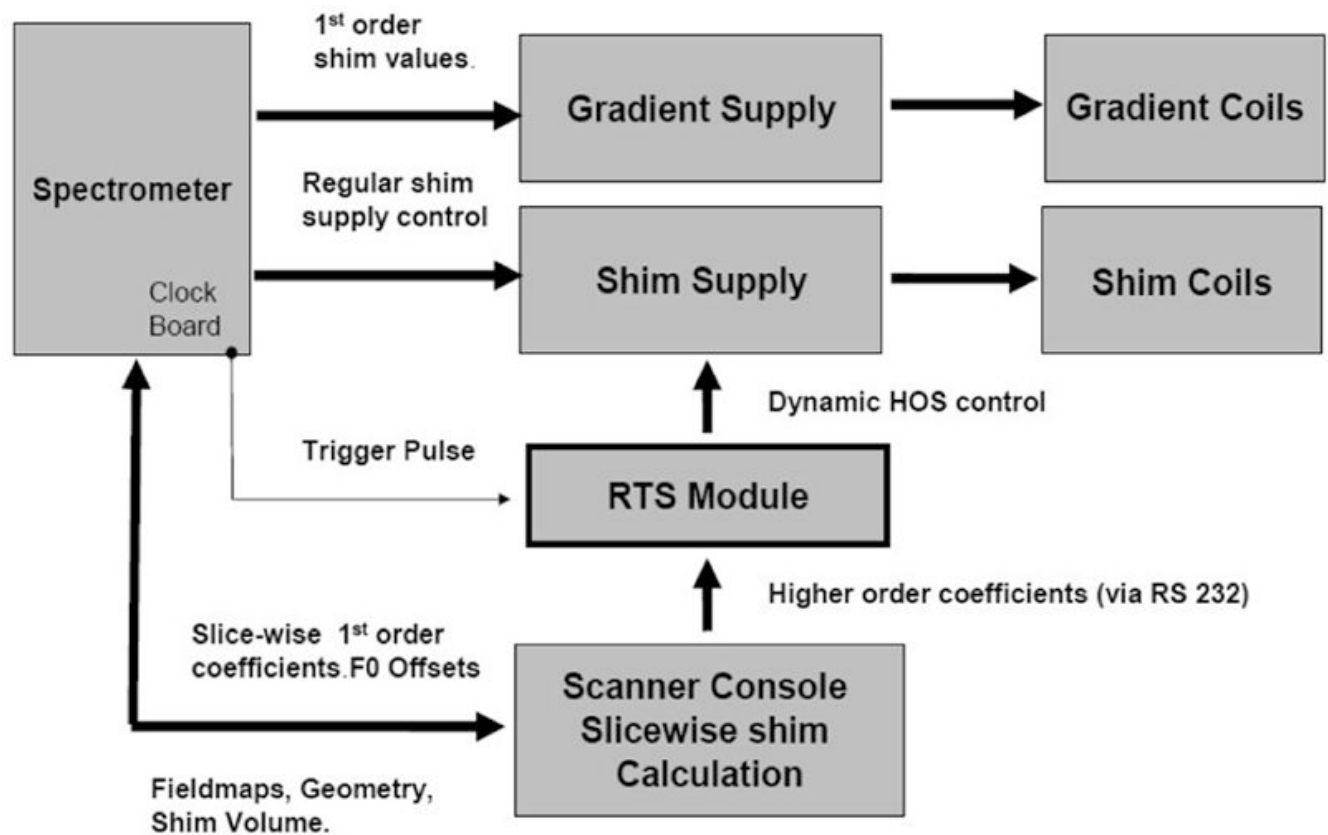

FIG. 1.

Workflow of DS. The regular higher order shim supply currents that add to the dynamic shim updates from the RTS may be set to zero during DS 
a Experimental (Axial)

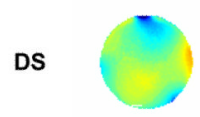

Slice

GS
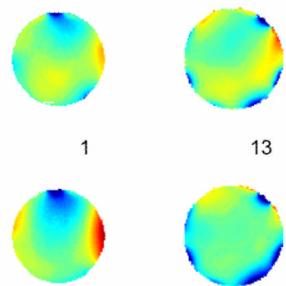

13

GS

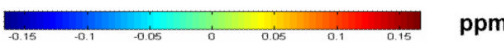

b Simulated (Axial)

DS

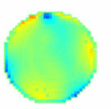

Slice
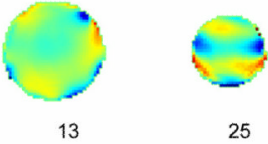

25

ppm c Slicewise standard deviation.

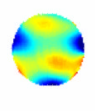

25

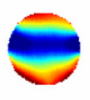

GS
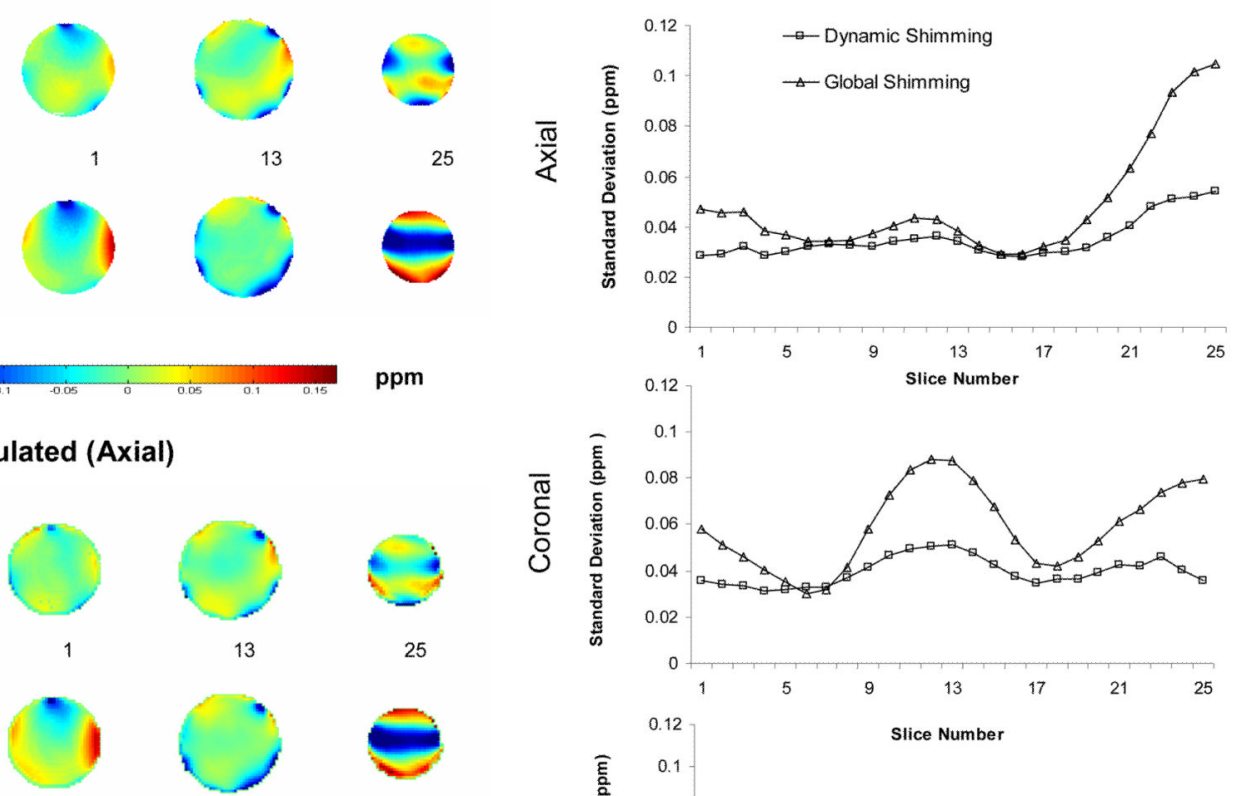

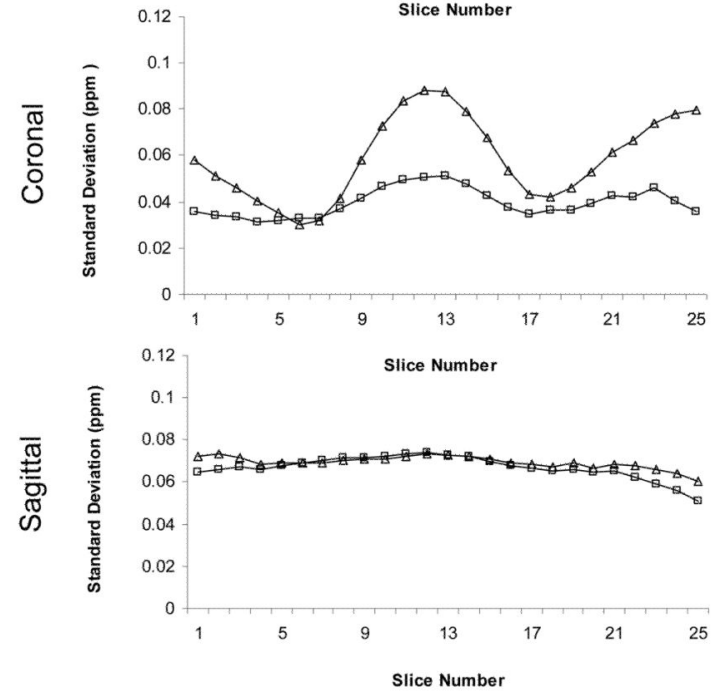

FIG.2.

Spherical doped water phantom fieldmaps in ppm of slices 1, 13 and 25 from 25 slice volumes after $2^{\text {nd }}$ order DS and $2^{\text {nd }}$ order image based static GS. (a) Fieldmaps from axial experiments. (b) Corresponding fieldmaps from simulations. Fieldmaps from experiments and simulations match up closely. Residual fields contain high degree of $3^{\text {rd }}$ order spatial harmonics as expected. (c) Slice-wise spherical phantom fieldmap standard deviations in 3 principal orientations; $2^{\text {nd }}$ order DS vs $2^{\text {nd }}$ order image based static GS. Dynamically shimmed fields had better homogeneity for all slices in all orientations. 


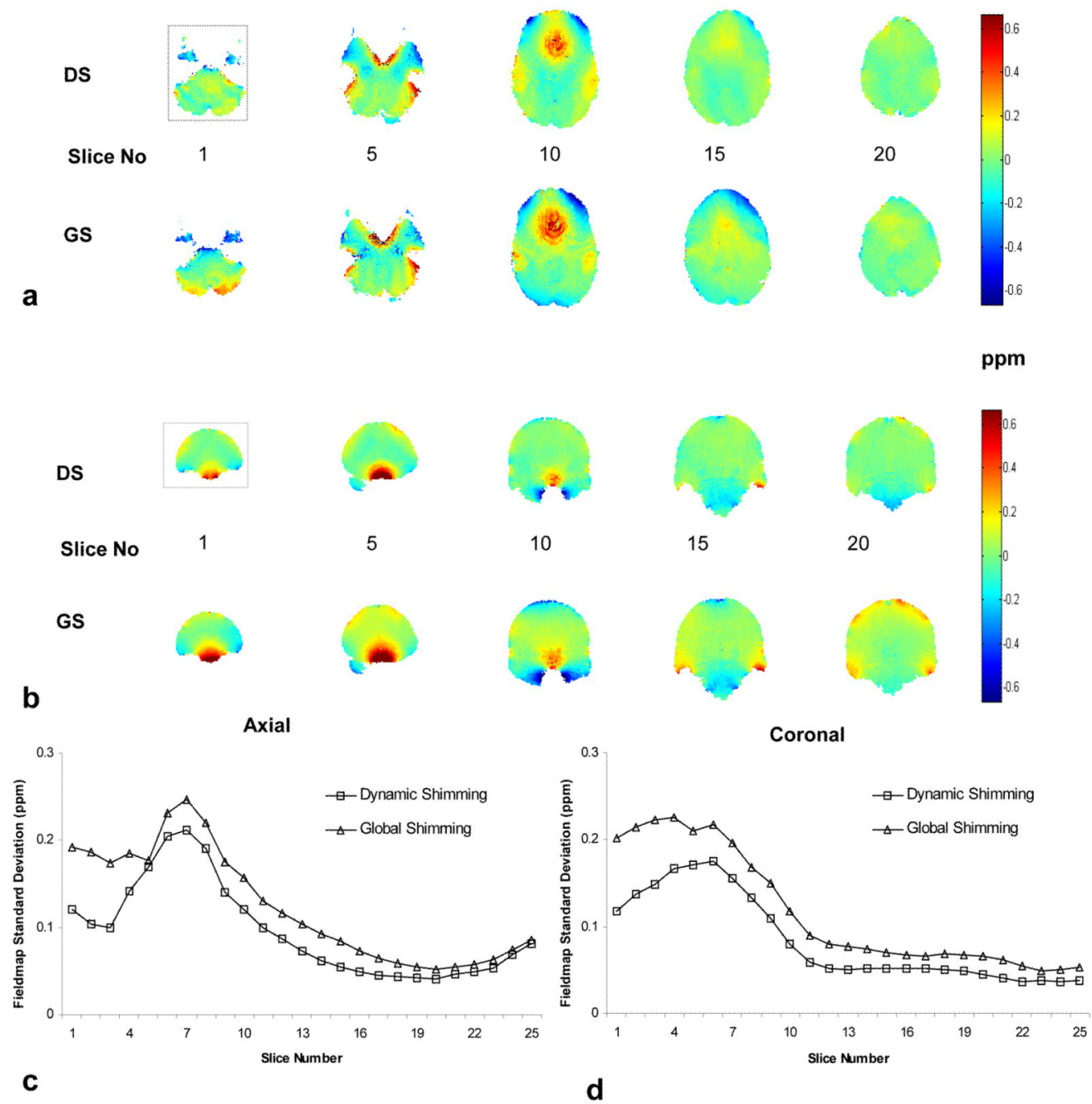

FIG. 3.

$\mathrm{B}_{0}$ fieldmaps in ppm of selected slices from 25 slice (a) axial and coronal (b) imaging volumes after $2^{\text {nd }}$ order DS and $2^{\text {nd }}$ order GS. Inplane ROIs are shown in the $1^{\text {st }} \mathrm{DS}$ fieldmap in both orientations, which extend to all slices. Slicewise f0 offsets observed for DS fieldmaps are specified in ppm. (c \& d) Slice-wise fieldmap standard deviations in ppm of the same imaging volumes within the ROIs. 
a

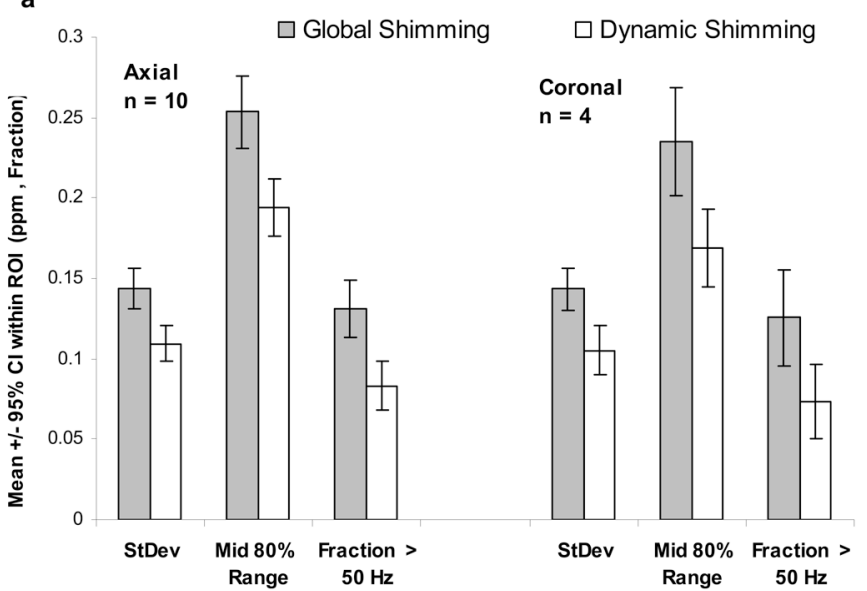

b

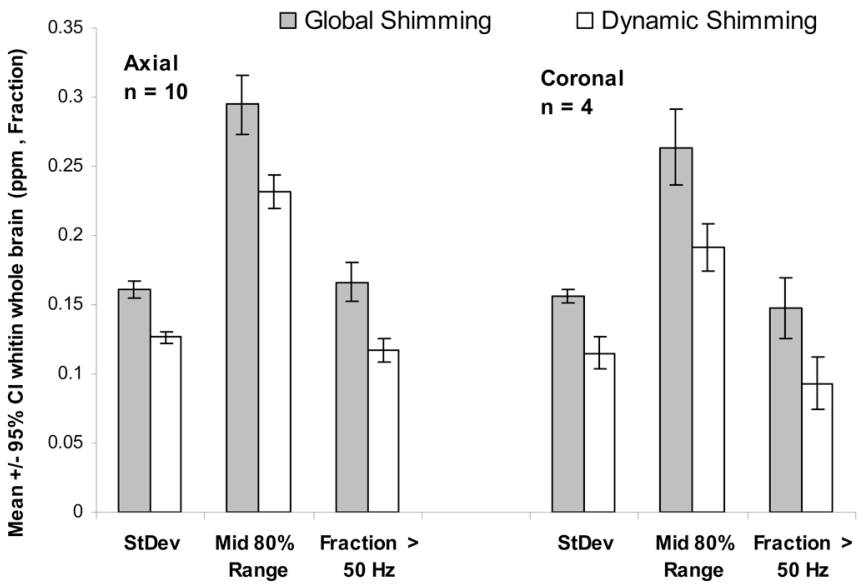

FIG. 4.

Overall $\mathrm{B}_{0}$ field homogeneity measures (Mean and $95 \%$ confidence intervals) after $2^{\text {nd }}$ order DS and $2^{\text {nd }}$ order GS (12 subjects, 10 axial scans, 4 coronal scans) calculated over (a) 25 slice brain imaging volume within the shim ROI and (b) within the whole brain.

Homogeneity measures include standard deviation (StDev), the central $80 \%$ pixel value range and the fraction of nonzero field value pixels greater than $50 \mathrm{~Hz}(0.167 \mathrm{ppm}$, corresponding to $\sim 2.5$ pixel inplane displacement at $19.4 \mathrm{~Hz} /$ pixel bandwidth). 


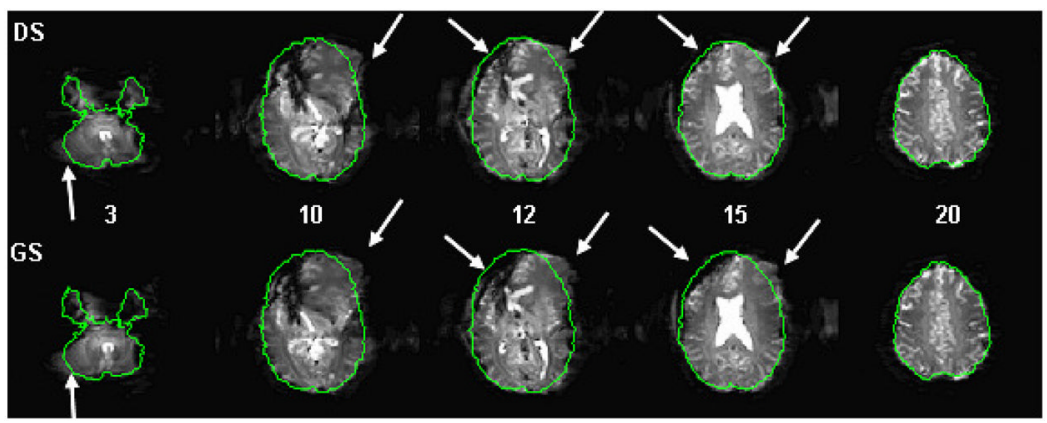

FIG. 5.

Single shot EPI geometric distortion comparison between $2^{\text {nd }}$ order DS and GS acquisitions for selected axial slices (slice numbers indicated in between). The green outlines derived from high bandwidth FFE images (not shown) serve as references. DS shows lesser distortions compared to GS, especially in the locations pointed out the arrows. 


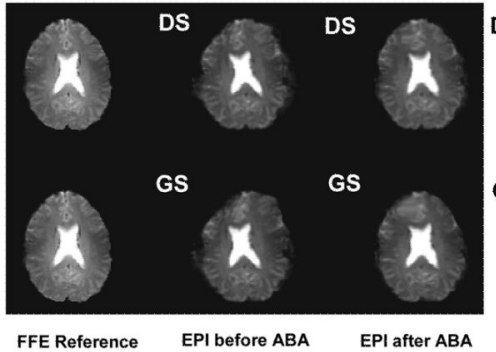

(a)

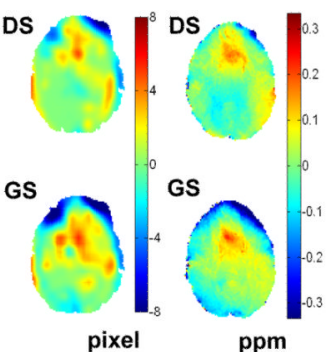

(b)

(c)

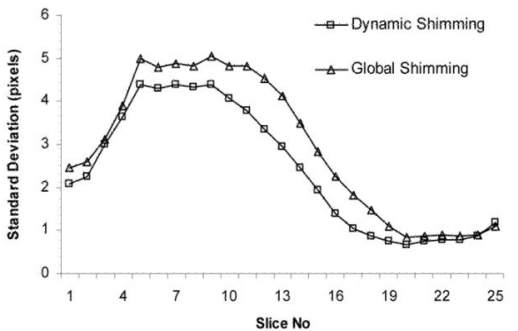

(d)

FIG. 6.

EPI deformation measurements. (a) Slice 15, DS and GS images, before and after ABA registration. (b) Pixel deformation map showing deformation from EPI to FFE reference in pixels. Positive values indicate pixel shifts to the right and negative values indicate shifts to the left. Phase encode direction was right-left. (c) Corresponding fieldmaps for the same slice in ppm. Pixel deviations in (b) match up closely with the underlying fieldmaps, validating the method and quantifying EPI distortion. (d) Slice-wise standard deviations of in-plane pixel displacement maps in global and dynamically shimmed EPI images. DS EPI images show much smaller pixel displacements in almost all slices compared to GS images. 


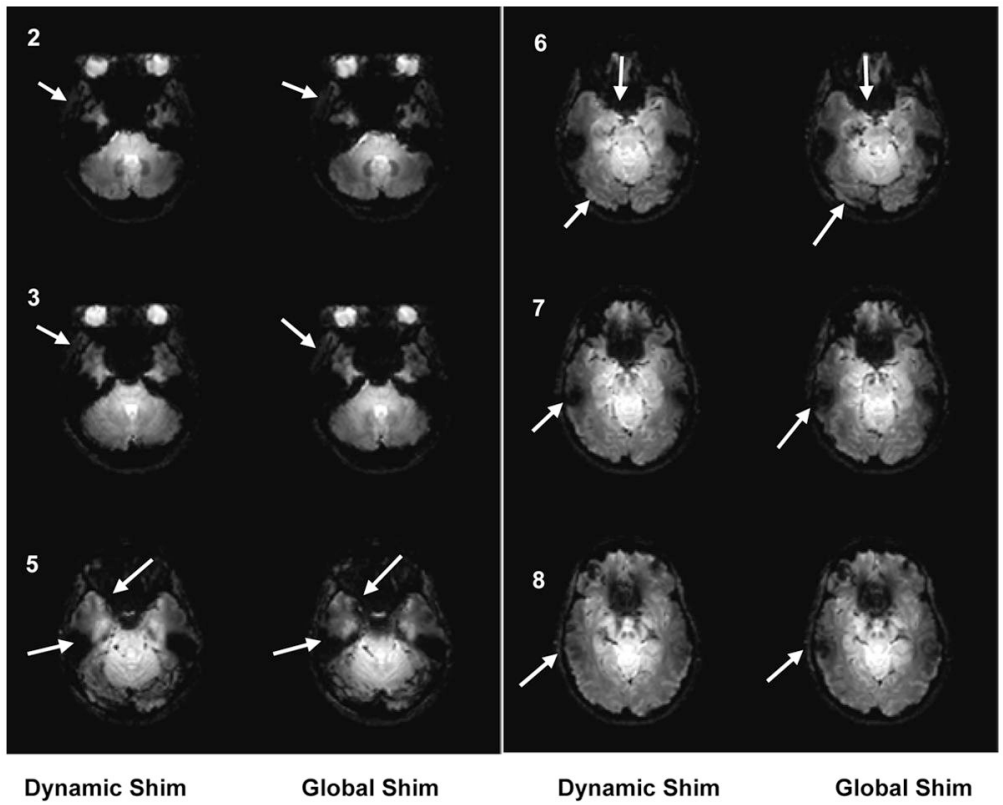

FIG. 7.

FFE signal loss comparison between DS and static GS acquisitions. DS shows higher signal recovery compared to static GS. Some areas with signal differences are demarcated by the arrows. Slice numbers indicated are from a 25 slice axial scan. 

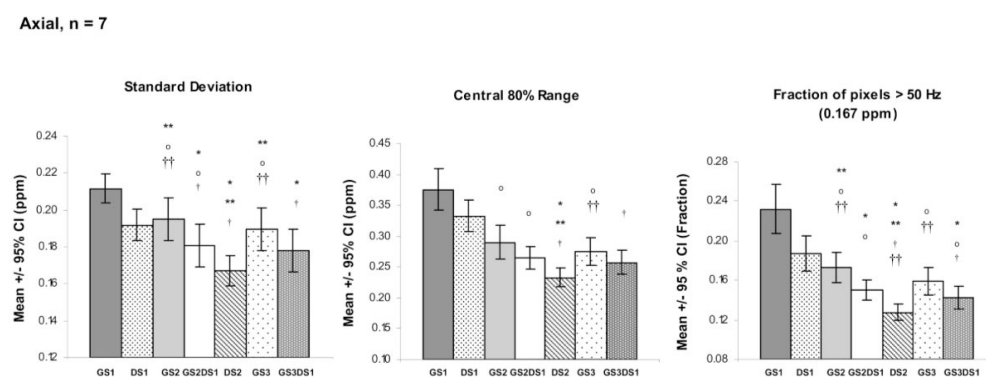

Coronal, $n=5$
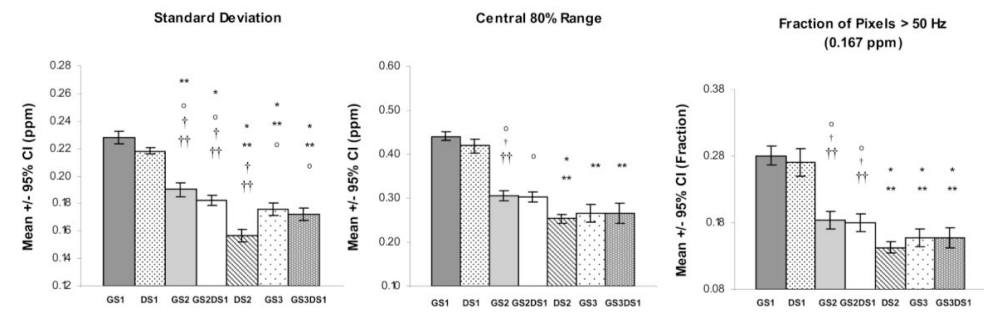

FIG. 8.

Results from Orderwise comparison of shims including $1^{\text {st }}, 2^{\text {nd }}$ and $3^{\text {rd }}$ order GS ( GS1, GS2 GS3), $1^{\text {st }}$ and $2^{\text {nd }}$ order DS (DS1, DS2) and combined $2^{\text {nd }}$ or $3^{\text {rd }}$ order GS and $1^{\text {st }}$ order DS (GS2DS1, GS3DS1) for 7 axial and 5 coronal subjects. Plots show Mean $+/-95 \%$ confidence interval of fieldmap standard deviation, the central $80 \%$ pixel value range and the fraction of nonzero field value pixels greater than $50 \mathrm{~Hz}$ over the whole brain. Markers $*, * *, \mathrm{o}, \dagger$ and $\dagger \dagger$ indicate significant differences with GS2, GS2DS1, DS2, GS3 and GS3DS1 respectively obtained from post-hoc paired t tests with bonferroni correction at $\alpha=$ 0.05 . 

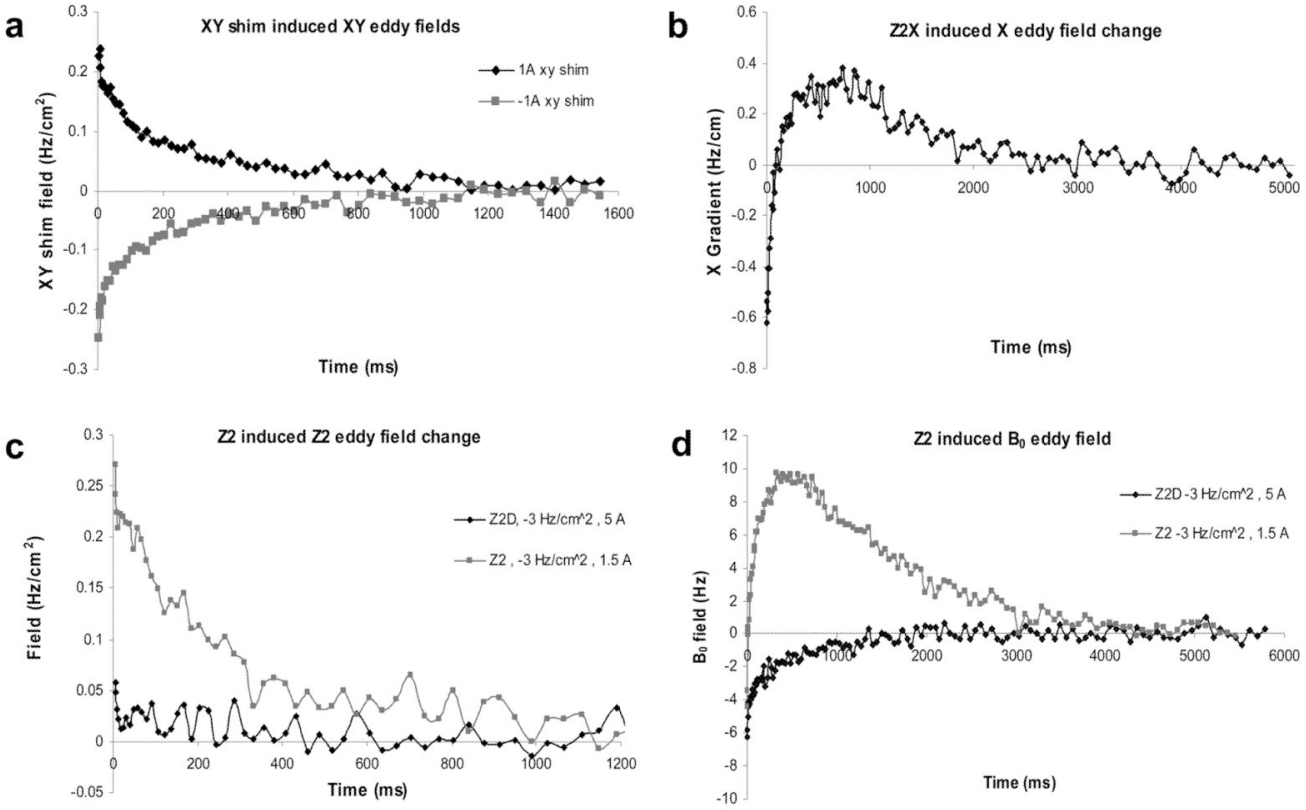

e

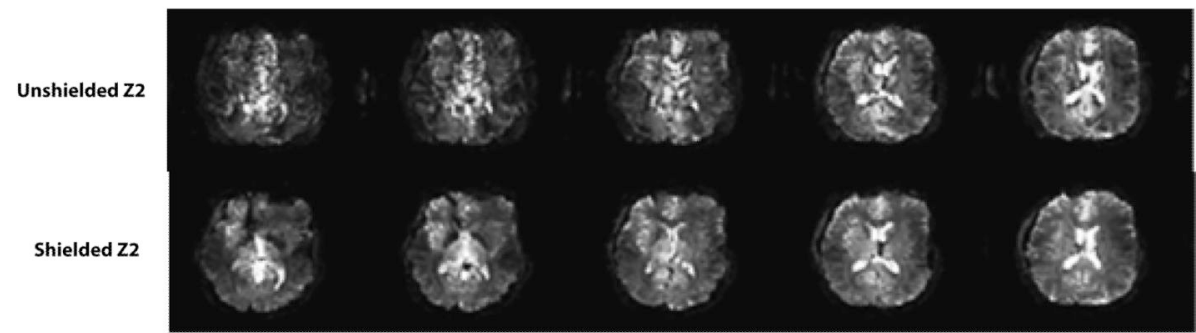

FIG.9.

Eddy current results (a): Eddy field decay after switching OFF of a XY shim pulse from 1A $\left(3.13 \mathrm{~Hz} / \mathrm{cm}^{2 /} \mathrm{A}\right)$ and $-1 \mathrm{~A}\left(-3.13 \mathrm{~Hz} / \mathrm{cm}^{2 /} \mathrm{A}\right)$. The shim component reduces to non significant levels around $1000 \mathrm{~ms}$ after shim turn off. (b) $\mathrm{X}$ shim eddy field produced by switching off the $3^{\text {rd }}$ order Z2X field from $1 \mathrm{~A}\left(-0.0155 \mathrm{~Hz} / \mathrm{cm}^{3 /} \mathrm{A}\right)(\mathbf{c} \&$ d) $\mathrm{Z} 2$ and B0 eddy fields produced by switching the unshielded Z2 $\left(-1.97 \mathrm{~Hz} / \mathrm{cm}^{2 /} \mathrm{A}\right)$ and $\mathrm{Z} 2 \mathrm{D}(-0.58 \mathrm{~Hz} /$ $\mathrm{cm}^{2 /} \mathrm{A}$ ) coils from $-3 \mathrm{~Hz} / \mathrm{cm}^{2}$ to $0 \mathrm{~Hz} / \mathrm{cm}^{2}$. Z2D coil is seen to produce much smaller field variations after switching than the unshielded Z2 coil.(e) Dynamically shimmed images using unshielded Z2 coil (top row) show significantly higher artifact levels due to eddy field variations than images acquired using Z2D coil (bottom row). 


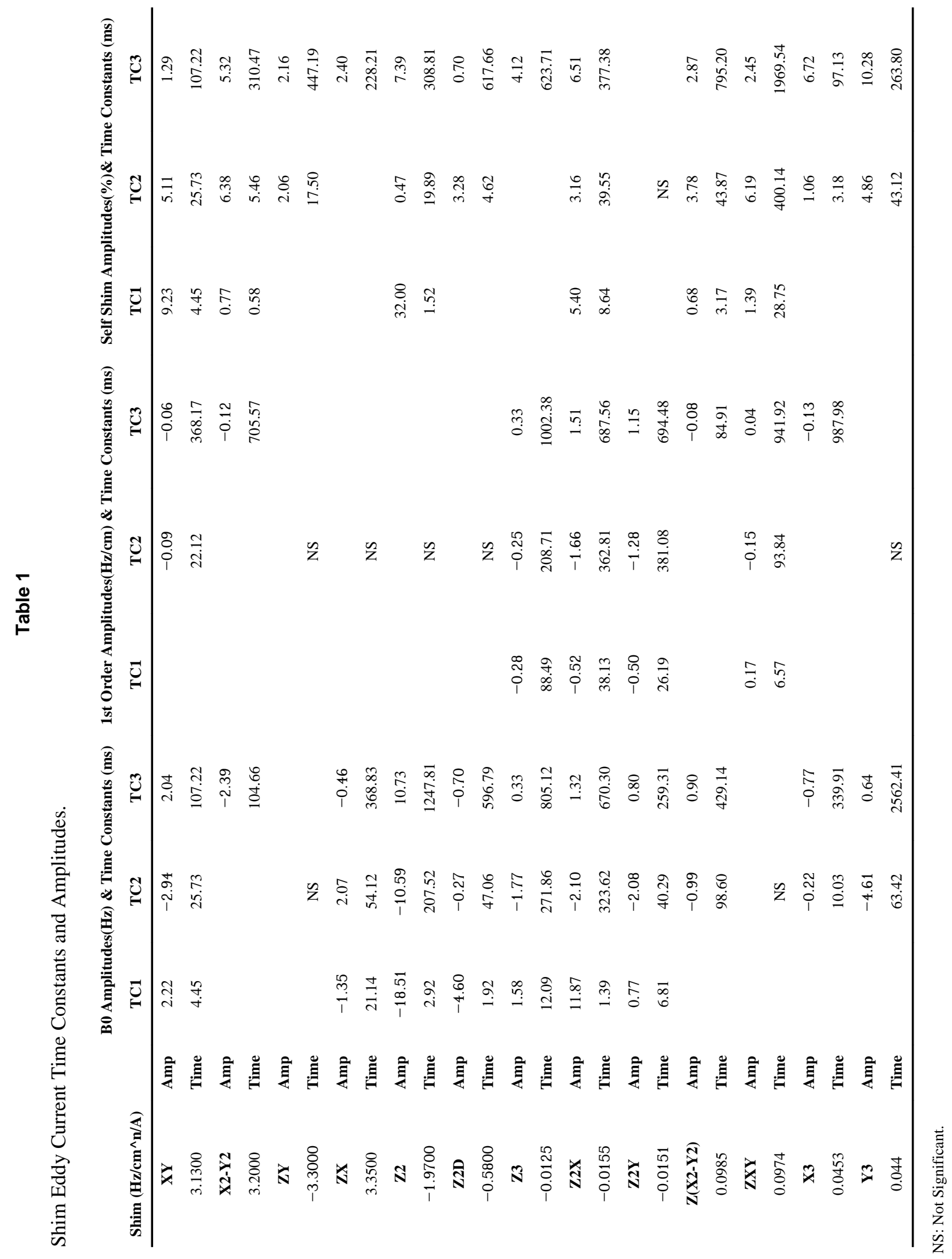

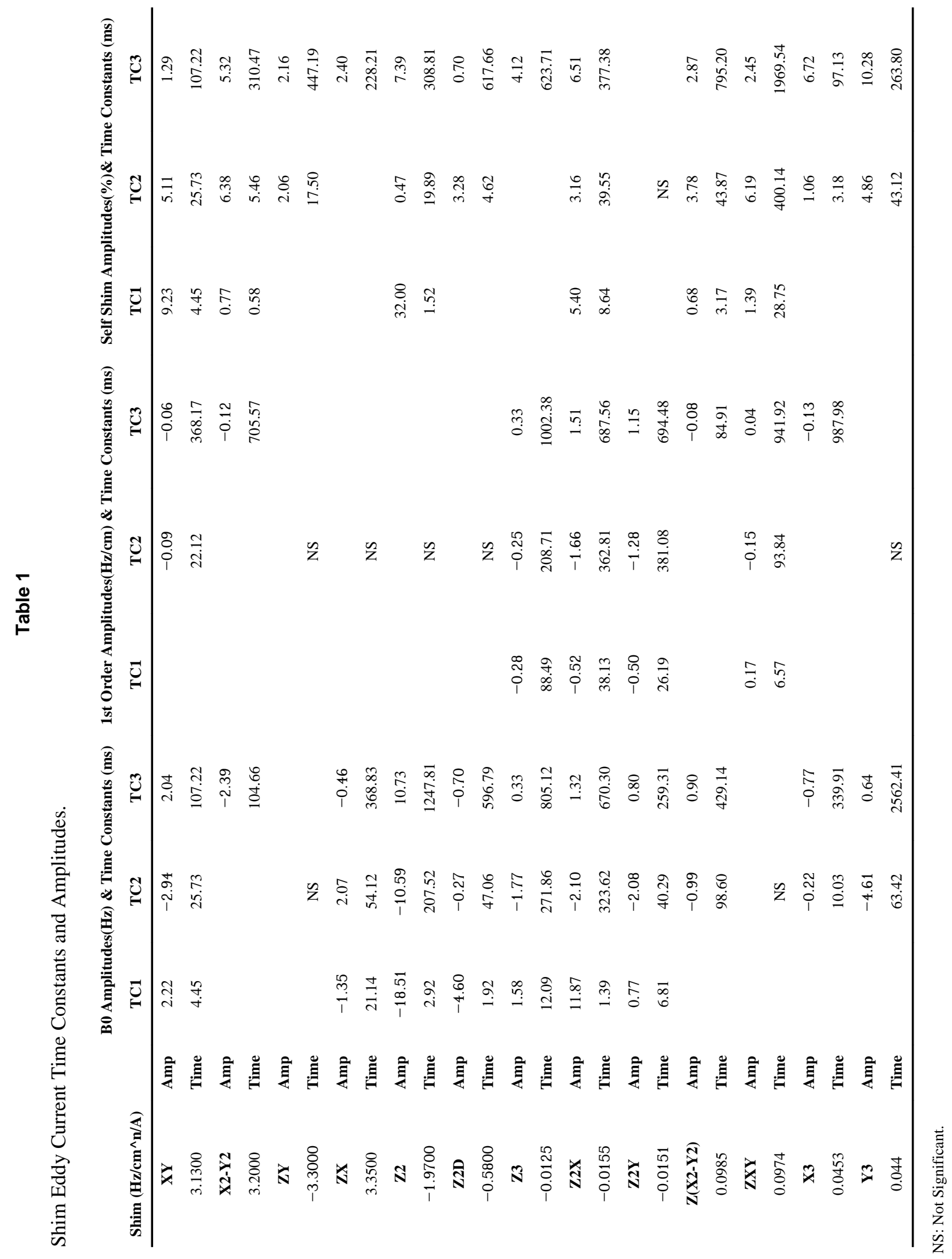

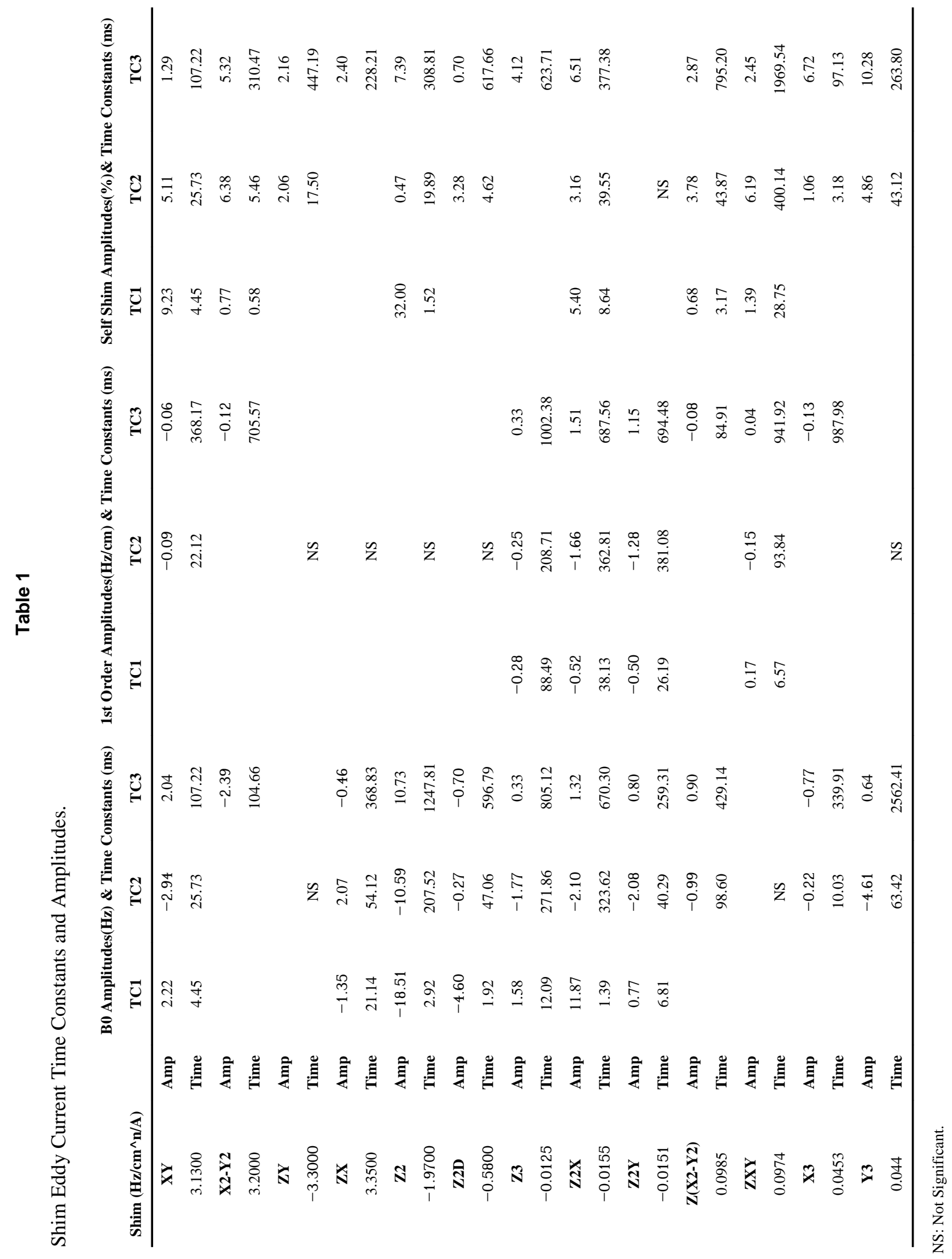

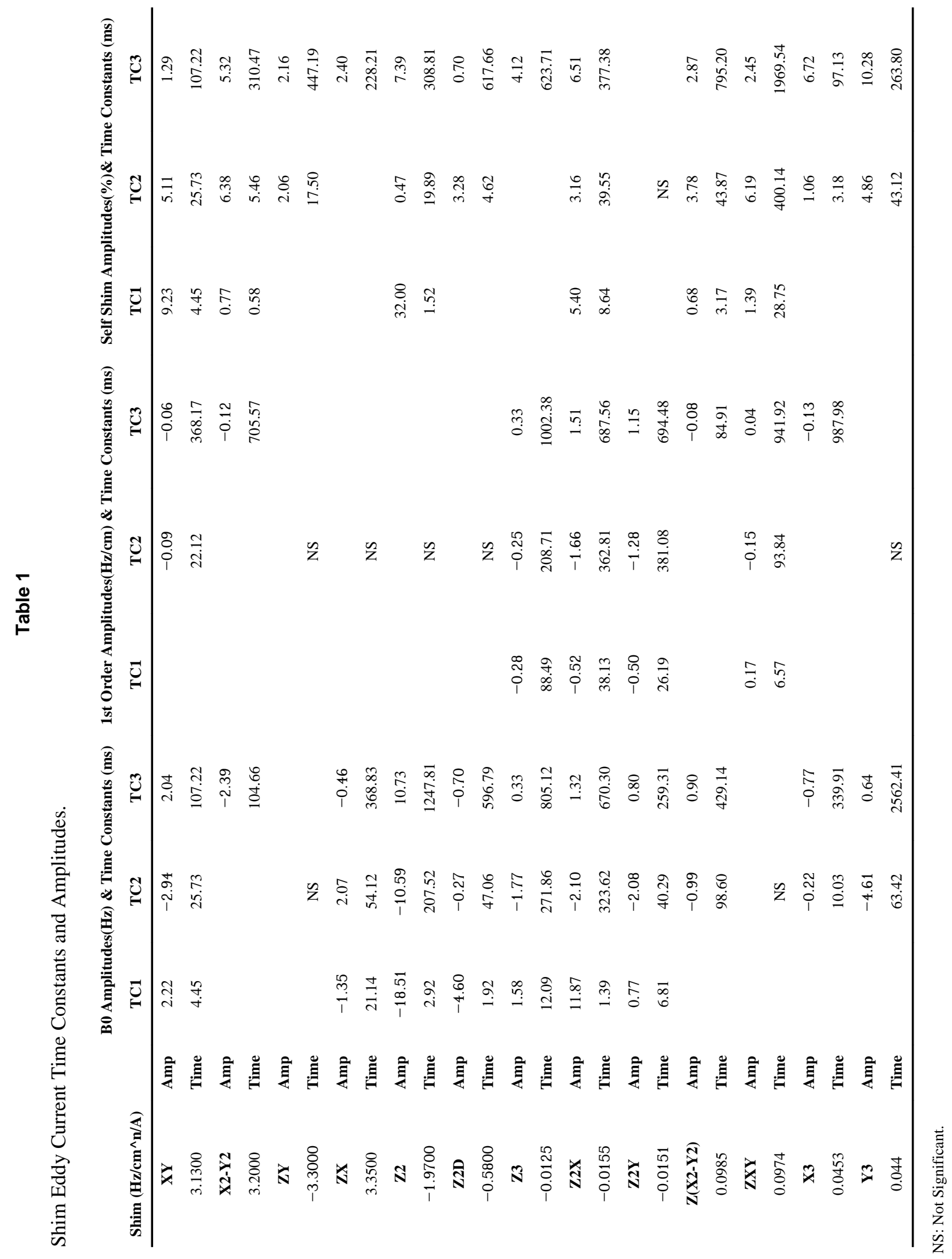

$$
\begin{aligned}
& \text { 方 }
\end{aligned}
$$

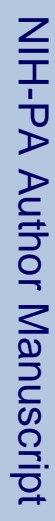

